\title{
Effect of painless STA on tooth extraction of elderly patients with periodontal diseases
}

\author{
LI FENG $^{1}$, HAIMING WANG ${ }^{2}$ and MING LIN $^{3}$ \\ ${ }^{1}$ Customer Service Management Office, Shandong University Affiliated to Jinan Central Hospital; \\ ${ }^{2}$ Department of Stomatology, Jinan Hospital; ${ }^{3}$ Department of Stomatology, Jinan Central \\ Hospital Affiliated to Shandong University, Jinan, Shandong 250013, P.R. China
}

Received October 11, 2017; Accepted December 15, 2017

DOI: $10.3892 / e t m .2018 .5776$

\begin{abstract}
This study compared the effects of tooth extractions under novel painless single tooth anesthesia (STA) and conventional anesthesia on elderly patients with periodontal diseases, and the impact of painless STA on clinical indicators of the patients. Elderly patients $(n=116)$ with periodontal diseases who were treated in Jinan Central Hospital were selected to undergo extraction operation on loose teeth; all the patients were randomly divided into two groups. The tooth extraction operations were performed after painless STA and cartridge anesthesia; the effects of the tooth extraction operations and the operation time were recorded; Visual Analogue Scale (VAS), Kuttner facial expression scale, Frankl treatment compliance scale and Houpt behavior rating scale were utilized to assess and record the clinical indicators of the patients during the operations; the impacts of the two anesthesia techniques on the effect of the tooth extraction of patients with periodontal diseases were comprehensively analyzed. Compared with conventional cartridge anesthesia, painless STA could effectively shorten the operation time $(\mathrm{P}<0.01)$; the Frankl treatment compliance score and Houpt behavior rating score of patients receiving painless STA were remarkably higher than those of patients receiving conventional cartridge anesthesia $(\mathrm{P}<0.01)$; during the operation, painless STA could effectively reduce the blood pressure and slow the heart rate by comparing with cartridge anesthesia $(\mathrm{P}<0.01, \mathrm{P}<0.05)$; the scores of STA and Kuttner facial expressions in patients receiving painless STA were significantly lower than those in patients receiving conventional cartridge anesthesia $(\mathrm{P}<0.01)$; after the operation, the incidence of adverse reactions, such as pain and swelling, in patients receiving painless STA was obviously decreased compared with that in patients receiving conventional cartridge anesthesia $(\mathrm{P}<0.01)$. The novel painless
\end{abstract}

Correspondence to: Dr Ming Lin, Department of Stomatology, Jinan Central Hospital Affiliated to Shandong University, 105 Jiefang Road, Jinan, Shandong 250013, P.R. China

E-mail: gm054x@163.com; linming1966@163.com

Key words: painless STA, periodontal diseases, tooth extraction, pain
STA can notably shorten the time of tooth extraction of elderly patients with periodontal diseases, reduce postoperative pain and swelling, increase the compliance of the operation, alleviate the impact of tooth extraction operation on the clinical indicators of the patients and relieve the pain of patients caused by the operation.

\section{Introduction}

With the improvement of people's living standard, increased importance has been attached to oral health, while the periodontal diseases seriously affect the quality of life of the elderly patients $(1,2)$. Tooth extraction operation is one of the most common operations for treating oral problems, and the pain caused by the operation or the unhealthy emotion of patients resulting from the pain often affects the quality and prognosis of the operation and even leads to severe adverse reactions $(3,4)$. The pain caused by the tooth extraction operation is usually relieved by local anesthesia, but the pain in the process of conventional cartridge anesthesia cannot be completely eliminated, and the effect is not good (5). Single tooth anesthesia (STA), which utilizes special injection technique and has an effect of minimal invasion, can minimize the pain of patients in the process of anesthesia and operation (6-8). There have been no studies on the impacts of STA and traditional cartridge anesthesia on the efficacy of tooth extraction operation on elderly patients with periodontal diseases yet. This study aimed to provide a theoretical basis for selection of appropriate anesthesia apparatus for elderly patients by analyzing and comparing the impacts of STA and cartridge anesthesia on the efficacy of tooth extraction of elderly patients with periodontal diseases, further offering a basis for promoting the use of STA.

\section{Materials and methods}

Instruments and materials. STA (MDSIN, USA), articaine hydrochloride and epinephrine tartrate injection (Pierre Rolland, Merignac, France), cartridge (Zhejiang Ouke Medical Instrument Co., Ltd.), physiological signal monitor (Shenzhen Wokang Medical Electronics Co., Ltd.), scalpels (Henan Chuangxin Medical Supplies, Co., Ltd.), absorbable sutures (Guangzhou Yuchao Medical Instrument Co., Ltd.); other 


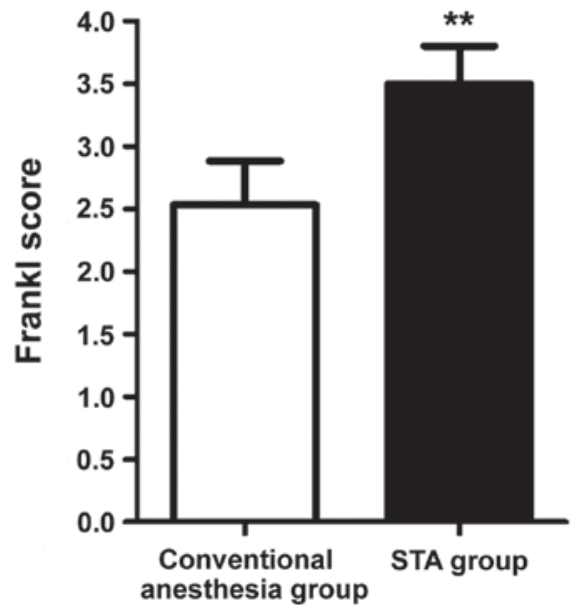

Figure 1. Evaluation of the patients' compliance with operation after different kinds of anesthesia using the Frankl treatment compliance scale; the score in STA group is obviously higher than that in conventional anesthesia group $\left({ }^{* *} \mathrm{P}<0.01\right)$.

relevant instruments and reagents are specified in the relevant parts. The study was approved by the Ethics Committee of Shandong University Affiliated Jinan Central Hospital (Shandong, China).

Research objects. All the patients of this study were selected from the elderly patients with periodontal diseases who were diagnosed and treated with tooth extraction in Jinan Central Hospital from May 2015 to May 2016. Inclusion criteria: 1) patients aged $>65$ years; 2) patients whose third molars were extracted, of which the operations were equally difficult; 3) patients without history of allergy to articaine hydrochloride and epinephrine tartrate injection; 4) patients without other diseases who were not given any drug during the one month period before the operation; 5) patients with similar judgment on pain. A total of 116 patients were enrolled, who were aged 65-78 years, including 61 males and 55 females; other wasting diseases were excluded from all the patients and they signed the informed consent. The above-mentioned patients had complete operation data and treatment protocol.

Surgical approaches. The enrolled patients were randomly divided into STA group $(n=58)$ and conventional anesthesia group $(n=58)$. The patients in STA group were anesthetized via an STA instrument with articaine hydrochloride and epinephrine tartrate injection at the periodontal ligament of the tooth to be extracted; the patients in conventional anesthesia group were anesthetized via a cartridge with articaine hydrochloride and epinephrine tartrate injection to the periodontal space; the same dose of anesthetic was applied to the two groups of patients. After anesthesia, both groups of patients underwent the following procedures: conventional disinfection, coverage of towel, tooth separation, bone removal and extraction of cacodontia.

Recording of tooth extraction operation time and patient's compliance. The time when the anesthesia was complete and the operation was started was regarded as the operation start time. During the operation, the compliance with operation was evaluated according to the patient's completion of surgeon's instructions and the Frankl treatment compliance scale. The compliance was divided into 4 grades, namely, complete non-compliance, partial non-compliance, partial compliance and complete compliance, which were marked as 1-4 points, respectively; the higher the score was, the better the patient's compliance would be. The time when the tooth socket was cleared up was regarded as the end of the operation; the operation time of each patient was recorded, compared and analyzed; the Houpt behavior rating scale was used to evaluate the smoothness of the operation; the smoothness was marked as 1-6 points, and the higher the score was, the smoother the operation would be.

Detection of clinical indicators. The physiological signal monitor was utilized to record the blood pressures and heart rates of the patients at $10 \mathrm{~min}$ before the anesthesia, $10 \mathrm{~min}$ after the start of the operation and $10 \mathrm{~min}$ after the end of the operation, respectively, which were compared and analyzed later.

Assessment of pain during the operation. The Visual Analogue Scale (VAS) was used to evaluate the pain of patients in the two groups during the tooth extraction operation. By means of enquiring from the patients, the severity of pain was evaluated as $0-10$ points, and the higher the score was, the more severe the pain caused by the operation would be. The Kuttner facial expression scale was applied to evaluate the facial expressions of the patients, which was marked at 0-6 points; the higher the score was, the more severe the pain during the operation would be.

Statistical analysis. All the data of this study are expressed as mean \pm standard deviation, and SPSS 19.0 software (SPSS Inc., Chicago, IL, USA) was used for data processing and analysis; t-test was used for comparison between groups, and analysis of variance was used for comparisons among multiple groups. After the examination by homogeneity test for variance, Bonferroni method was utilized for pairwise comparison in case of homogeneity of variance, and Welch method in case of heterogeneity of variance; Dunnett's T3 test was applied to multiple comparisons; $\mathrm{P}<0.05$ was considered to indicate a statistically significant difference.

\section{Results}

Impact of different anesthesia techniques on patients' compliance with operation and the operation effect. The Frankl treatment compliance scale was used to evaluate the patients' compliance with operation after different kinds of anesthesia. As shown in Fig. 1, the Frankl treatment compliance score in STA group was obviously higher than that in conventional anesthesia group, and the difference was statistically significant $(\mathrm{P}<0.01)$. The Houpt behavior rating scale was applied to evaluate the smoothness of the operation. As shown in Fig. 2, the Houpt behavior rating score in STA group was remarkably higher than that in conventional anesthesia group, and the difference was statistically significant $(\mathrm{P}<0.01)$. The total time of the tooth extraction operation in the two groups is 


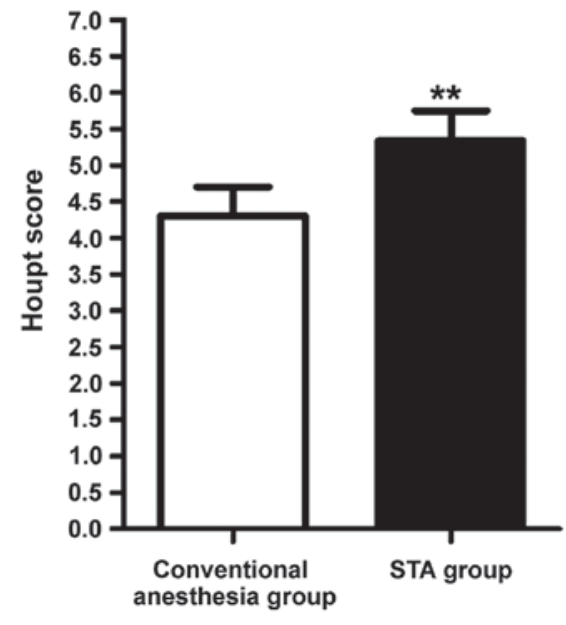

Figure 2. Evaluation of the smoothness of the operation using the Houpt behavior rating scale; the score in STA group is remarkably higher than that in conventional anesthesia group $\left.{ }^{* *} \mathrm{P}<0.01\right)$.

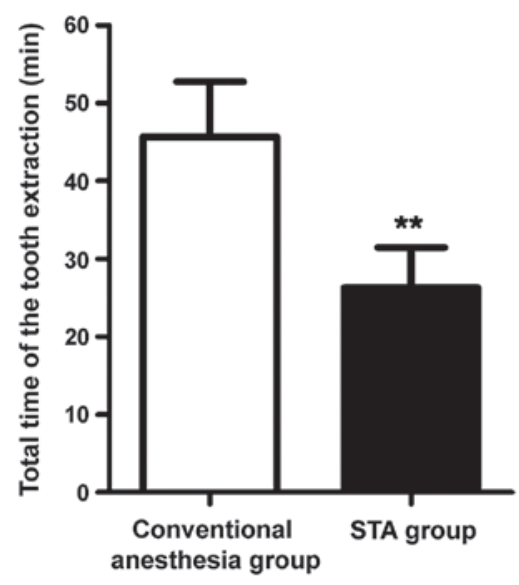

Figure 3. Total time of the tooth extraction; the operation time in STA group is shorter than that in conventional anesthesia group $(* * \mathrm{P}<0.05)$.

Table I. Occurrence of pain and swelling in the periodontal region after the operation.

\begin{tabular}{lccc}
\hline Group & $\begin{array}{c}\text { Periodontal } \\
\text { pain (n) }\end{array}$ & $\begin{array}{c}\text { Periodontal } \\
\text { swelling (n) }\end{array}$ & $\begin{array}{c}\text { Incidence } \\
\text { of adverse } \\
\text { reactions (\%) }\end{array}$ \\
\hline Conventional anesthesia & 2 & 3 & 8.6 \\
STA & 6 & 13 & 32.8 \\
P-value & $<0.01$ & $<0.01$ & $<0.01$ \\
t-value & 0.962 & 1.037 & 3.296 \\
\hline
\end{tabular}

shown in Fig. 3; the operation time in STA group was shorter than that in conventional anesthesia group $(\mathrm{P}<0.05)$.

Impacts of different anesthesia techniques on patients' blood pressures and heart rates. The physiological signal monitoring system was utilized to record the blood pressures and heart rates of the patients in the two groups at $10 \mathrm{~min}$ before the anesthesia, 10 min after the start of the operation and 10 min after the end of the operation, respectively. As shown in Figs. 4 and 5, there were no statistically significant differences in blood pressures and heart rates between the two groups before the anesthesia $(\mathrm{P}>0.05)$; at $10 \mathrm{~min}$ after the start of the operation, the blood pressure and heart rate in conventional anesthesia group were notably higher than those in STA group, and the differences were statistically significant $(\mathrm{P}<0.01)$; at $10 \mathrm{~min}$ after the end of the operation, the blood pressure and heart rate in conventional anesthesia group were decreased, but they were still higher than those in STA group $(\mathrm{P}<0.05)$.

Assessment of pain during the operation. VAS and Kuttner facial expression scale were used to evaluate the pain of the patients during the operation. As shown in Fig. 6, the scores of VAS and Kuttner facial expressions in STA group were obviously lower than those in conventional anesthesia group, and the differences were statistically significant $(\mathrm{P}<0.01)$.

Occurrence of adverse reactions after the operation. The adverse reactions of the patients, including pain and swelling in the periodontal region, were recorded after the operation. As shown in Table I, the incidences of pain, swelling and other adverse reactions in STA group were obviously decreased compared with those in conventional anesthesia group, and the differences were statistically significant $(\mathrm{P}<0.01)$.

\section{Discussion}

As increasing attention has been paid to oral health, the number of dental operations is increasing year by year, and the anesthetic effect in the process of operation often influences the smoothness and effect of the operation (9-11). Conventional cartridge anesthesia can trigger fear in patients and decrease their cooperation during the operation because it cannot completely eliminate the pain of the patients during the operation, and there is a certain degree of pain produced during the anesthesia $(12,13)$. Conventional anesthesia technique may cause a certain degree of injury to the periodontal region, thus leading to discomfort of gingival distending pain in patients after the operation; however, the novel STA process belongs to a minimally invasive procedure, which may minimize the area of trauma, alleviate the patient's fear to the operation and make the operation smoother $(14,15)$. As special populations, the elderly patients usually have a poor operative effect due to inappropriate anesthesia technique, influencing the prognosis (16). Therefore, it is very important for the tooth extraction operation for elderly patients with periodontal disease to evaluate and choose proper anesthesia techniques.

In this study, the compliance of the patients, smoothness of the operation, severity of patients' pain during the operation and the impacts of the operation on the patients' heart rate and blood pressure were compared by comparing the effects of conventional cartridge anesthesia and STA on tooth extraction. The results showed that local anesthesia via STA instrument can stabilize the patients' emotions and alleviate their fear during the operation; compared with cartridge anesthesia, STA can effectively relieve the patients' pain during the operation $(\mathrm{P}<0.01)$. It was also found in this study that compared with that by conventional anesthesia, the local 
A

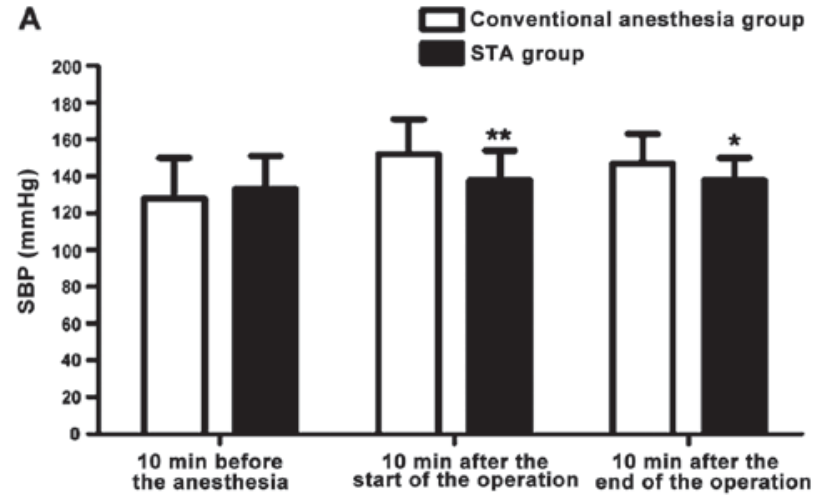

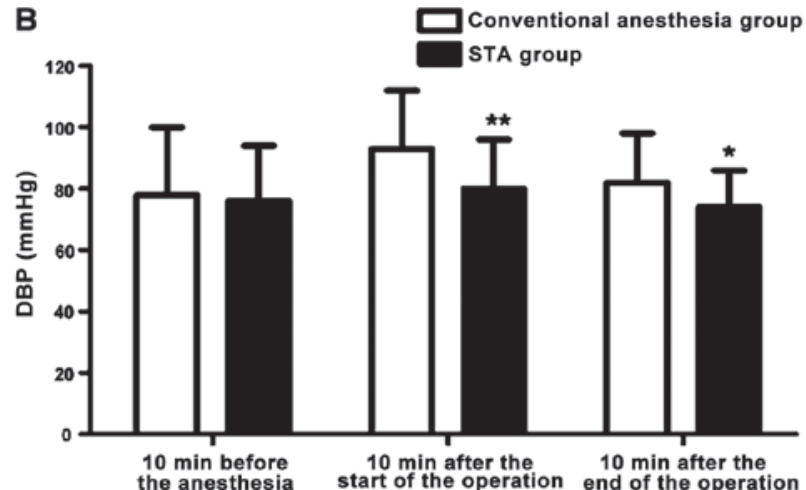

Figure 4. Variation trends of blood pressures in each group. (A) The values of systolic blood pressure (SBP), and (B) the values of diastolic blood pressure (DBP); there is no statistically significant difference in blood pressure between the two groups before the anesthesia (P>0.05); at 10 min after the start of the operation, the blood pressure in STA group is notably lower than that in conventional anesthesia group $\left({ }^{* *} \mathrm{P}<0.01\right)$; at 10 min after the end of the operation, the blood pressure in conventional anesthesia group is higher than that in STA group $\left({ }^{*} \mathrm{P}<0.05\right)$.

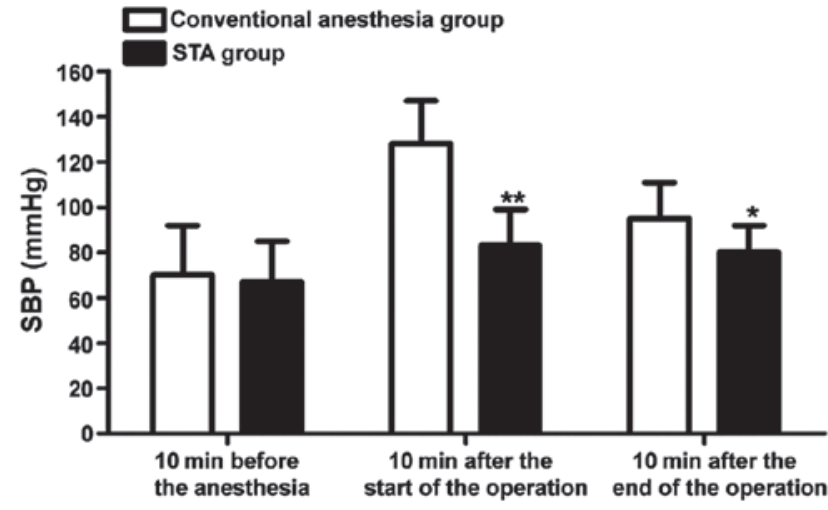

Figure 5. Variation trends of heart rates in each group. There is no statistically significant difference in heart rate between the two groups before the anesthesia $(\mathrm{P}>0.05)$; at $10 \mathrm{~min}$ after the start of the operation, the heart rate in STA group is notably lower than that in conventional anesthesia group $\left({ }^{* *} \mathrm{P}<0.01\right)$; at $10 \mathrm{~min}$ after the end of the operation, the heart rate in conventional anesthesia group is higher than that in STA group $\left({ }^{*} \mathrm{P}<0.05\right)$.
A

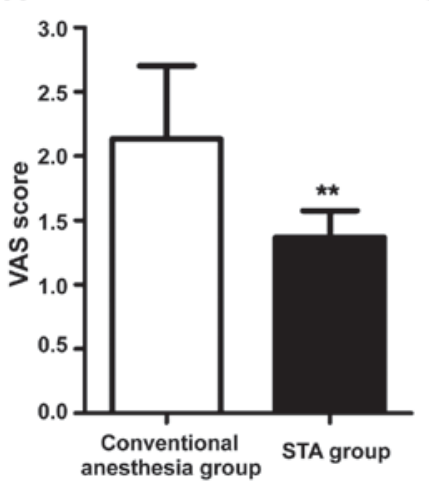

B

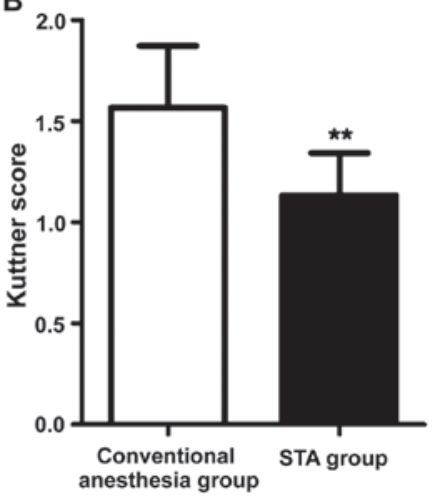

Figure 6. Assessment of pain during the operation: (A) the scores of VAS, and (B) the scores of Kuttner facial expressions; the scores of VAS and Kuttner facial expressions in STA group are obviously lower than those in conventional anesthesia group $\left({ }^{* *} \mathrm{P}<0.01\right)$.

anesthesia by STA instrument can remarkably increase the Frankl treatment compliance score and Houpt behavior rating score in the process of operation $(\mathrm{P}<0.01)$. It indicates that STA can improve the patients' compliance during the operation and the smoothness of the operation.

Arapostathis et al (17) found that compared with conventional oral anesthesia techniques, STA can effectively reduce the crying, cooperation and other behaviors of the children during the tooth extraction and shorten the operation time. STA has many characteristics, such as small size, simple and convenient procedures and high safety, of which the procedures can be completed by most of the dentists independently. The fluctuations of blood pressure and heart rate of the patients who underwent tooth extraction after local anesthesia via STA instrument were obviously lower than those of patients who underwent tooth extraction after local anesthesia via cartridge $(\mathrm{P}<0.01)$. STA can mitigate the fear of the patients during the operation, has a small trauma on the patients and causes small variations in blood pressure and heart rate, thus having a significant advantage in safety compared with cartridge anesthesia (18).

The study of Jia et al (19) discovered that local anesthesia by STA instrument had no influence on the blood pressure and heart rate of children patients who received the tooth extraction operation, which is consistent with the result of this study. STA can significantly decrease the scores of VAS and Kuttner facial expressions of the patients during the operation, and mitigate the pain of patients caused by the operation and lower the incidences of pain, swelling and other adverse reactions in the periodontal region after the operation. The above results indicate that STA can decrease the bad experiences of the patients during the operation and improve their acceptability to the operation. However, there are limited local anesthetics suitable for STA, and only articaine hydrochloride and epinephrine tartrate injection can be used; moreover, patients with allergy or intolerance to the drug cannot be anesthetized by STA (20).

In conclusion, the tooth extraction operation under STA can effectively lower the severity of pain of elderly patients with periodontal disease during the operation, improve the patients' compliance with the operation and shorten the operation time, without influencing the effect of tooth extraction operation on patients' blood pressure and heart rate and with good prognosis; therefore, as a new type of anesthesia technique, STA is worth publicizing and applying in dental operations. 


\section{Acknowledgements}

Not applicable.

\section{Funding}

Not applicable.

\section{Availability of data and materials}

The datasets used and/or analyzed during the present study are available from the corresponding author on reasonable request.

\section{Authors' contributions}

Not applicable.

\section{Ethics approval and consent to participate}

The study was approved by the Ethics Committee of Shandong University Affiliated Jinan Central Hospital (Shandong, China). Signed written informed consents were obtained from the patients and/or guardians.

\section{Consent for publication}

Not applicable.

\section{Competing interests}

The authors declare that they have no competing interests.

\section{References}

1. Nazir MA: Prevalence of periodontal disease, its association with systemic diseases and prevention. Int J Health Sci (Qassim) 11: $72-80,2017$.

2. Silva VO, Lobato RV, Andrade EF, de Macedo CG, Napimoga JT, Napimoga MH, Messora MR, Murata RM and Pereira LJ: $\beta$-Glucans (Saccharomyces cereviseae) reduce glucose levels and attenuate alveolar bone loss in diabetic rats with periodontal disease. PLoS One 10: e0134742, 2015.

3. Jiang H, Zhang Y, Xiong X, Harville EW, Karmin O and Qian X: Salivary and serum inflammatory mediators among pre-conception women with periodontal disease. BMC Oral Health 16: 131, 2016.

4. Grønkjær LL: Periodontal disease and liver cirrhosis: A systematic review. SAGE Open Med 3: 2050312115601122,2015

5. Kalakonda B, Koppolu P, Baroudi K and Mishra A: Periodontal systemic connections - novel associations - A review of the evidence with implications for medical practitioners. Int J Health Sci (Qassim) 10: 293-307, 2016.

6. Yamamoto N, Oshima M, Tanaka C, Ogawa M, Nakajima K, Ishida K, Moriyama K and Tsuji T: Functional tooth restoration utilising split germs through re-regionalisation of the toothforming field. Sci Rep 5: 18393, 2015.
7. Hosey MT, Donaldson AN, Huntington C, Liossi C, Reynolds PA, Alharatani R and Newton JT: Improving access to preparatory information for children undergoing general anaesthesia for tooth extraction and their families: Study protocol for a Phase III randomized controlled trial. Trials 15: 219, 2014.

8. Taylor PM, Hoare HR, de Vries A, Love EJ, Coumbe KM, White KL and Murrell JC: A multicentre, prospective, randomised, blinded clinical trial to compare some perioperative effects of buprenorphine or butorphanol premedication before equine elective general anaesthesia and surgery. Equine Vet J 48: 442-450, 2016.

9. Kuroshima S, Mecano RB, Tanoue R, Koi K and Yamashita J: Distinctive tooth-extraction socket healing: Bisphosphonate versus parathyroid hormone therapy. J Periodontol 85: 24-33, 2014.

10. Lu P, Gong Y, Chen Y, Cai W and Sheng J: Safety analysis of tooth extraction in elderly patients with cardiovascular diseases. Med Sci Monit 20: 782-788, 2014.

11. Sun Z, Herring SW, Tee BC and Gales J: Alveolar ridge reduction after tooth extraction in adolescents: An animal study. Arch Oral Biol 58: 813-825, 2013.

12. Furuta M, Shimazaki Y, Takeshita T, Shibata Y, Akifusa S, Eshima N, Kiyohara Y, Ninomiya T, Hirakawa Y, Mukai N, et al: Gender differences in the association between metabolic syndrome and periodontal disease: The Hisayama Study. J Clin Periodontol 40: 743-752, 2013.

13. Ricardo AC, Athavale A, Chen J, Hampole H, Garside D, Marucha P and Lash JP: Periodontal disease, chronic kidney disease and mortality: Results from the third National Health and Nutrition Examination Survey. BMC Nephrol 16: 97, 2015.

14. Oshima M, Inoue K, Nakajima K, Tachikawa T, Yamazaki H, Isobe T, Sugawara A, Ogawa M, Tanaka C, Saito M, et al: Functional tooth restoration by next-generation bio-hybrid implant as a bio-hybrid artificial organ replacement therapy. Sci Rep 4: 6044, 2014.

15. Gazal G, Alharbi R, Fareed WM, Omar E, Alolayan AB, Al-Zoubi $\mathrm{H}$ and Alnazzawi AA: Comparison of onset anesthesia time and injection discomfort of $4 \%$ articaine and 2\% mepivacaine during teeth extractions. Saudi J Anaesth 11: 152-157, 2017.

16. Jiang H, Su Y, Xiong X, Harville E, Wu H, Jiang Z and Qian X: Prevalence and risk factors of periodontal disease among preconception Chinese women. Reprod Health 13: 141, 2016.

17. Arapostathis KN, Dabarakis NN, Coolidge T, Tsirlis A and Kotsanos N: Comparison of acceptance, preference, and efficacy between jet injection INJEX and local infiltration anesthesia in 6 to 11 year old dental patients. Anesth Prog 57: 3-12, 2010.

18. Beneito-Brotons R,Peñarrocha-Oltra D, Ata-Ali J and Peñarrocha M: Intraosseous anesthesia with solution injection controlled by a computerized system versus conventional oral anesthesia: A preliminary study. Med Oral Patol Oral Cir Bucal 17: e426-e429, 2012.

19. Jia ZM, Hao HN, Huang ML, Ma DF, Jia XL and Ma B: Influence of dexmedetomidine to cognitive function during recovery period for children with general anesthesia. Eur Rev Med Pharmacol Sci 21: 1106-1111, 2017.

20. Negrato CA, Tarzia O, Jovanovič L and Chinellato LE: Periodontal disease and diabetes mellitus. J Appl Oral Sci 21: 1-12, 2013. 